\title{
TWO EXAMPLES IN PROXIMITY SPACES
}

\author{
P. L. SHARMA
}

ABSTR ACT. Two examples of Lo-spaces are given. The first is an example of a Lo-space in which not every ultrafilter is contained in a cluster. In the Lo-space of the second example, each ultrafilter is contained in a cluster, and yet not every maximal bunch is a cluster.

It is well known that in Efremovič proximity spaces each maximal bunch is a cluster and also each ultrafilter is contained in a unique cluster. In Example 1 we construct a Lo-space in which not every ultrafilter is contained in a cluster and, consequently, in that space, not every maximal bunch is a cluster. Surprisingly there also exist Lo-spaces in which every ultrafilter is contained in a cluster and still there are maximal bunches which are not clusters. One such space is outlined in Example 2.

We shall be using the terminology of [1], some of which is given below.

Let $\delta$ be a binary relation on the power set of a nonempty set $X$. Consider the following axioms:

$$
\begin{aligned}
& \left(\mathrm{P}_{0}\right)(\{x\},\{y\}) \in \delta \text { implies } x=y ; \\
& \left(\mathrm{P}_{1}\right)(\phi, x) \notin \delta \text {; } \\
& \left(\mathrm{P}_{2}\right)(A, B) \in \delta \text { implies }(B, A) \in \delta ; \\
& \left(\mathrm{P}_{3}\right) A \cap B \neq \varnothing \text { implies }(A, B) \in \delta ; \\
& \left(\mathrm{P}_{4}\right)(A, B \cup C) \in \delta \text { if and only if }(A, B) \in \delta \text { or }(A, C) \in \delta ; \\
& \left(\mathrm{P}_{5}\right)(A, B) \in \delta \text { and }(\{b\}, C) \in \delta \text { for each } b \in B \text { implies }(A, C) \in \delta ; \\
& \left(\mathrm{P}_{6}\right)(A, B) \notin \delta \text { implies that there exists a subset } E \text { of } X \text { such that }
\end{aligned}
$$
$(A, E) \notin \delta$ and $(X-E, B) \notin \delta$.

(i) $\delta$ satisfying $\left(\mathrm{P}_{1}-\mathrm{P}_{5}\right)$ is called a Looproximity.

(ii) $\delta$ satisfying $\left(\mathrm{P}_{1}-\mathrm{P}_{4}\right)$ and $\left(\mathrm{P}_{6}\right)$ is called an Efremovič proximity (or EF proximity).

(iii) $\delta$ satisfying $\left(\mathrm{P}_{0}\right)$ is called separated. Clearly every EF proximity is a Lo-proximity but not conversely. If $\delta$ is a Lo-proximity (EF proximity) on $X$, then the pair $(X, \delta)$ is called a Lospace (resp. EF space).

A topological space $X$ is $R_{0}$ if and only if for each $x \in X$ and each neighborhood $G$ of $X$, we have $\overline{\{x\}} \subset G$. A Lo-proximity $\delta$ on a set $x$ induces an $R_{0}$ topology on $X$ via the Kuratowski closure operator given by $\bar{A}=\{x \in X:(\{x\}, A) \in \delta\}$.

Received by the editors September 30, 1974. AMS (MOS) subject classifications (1970). Primary 54E 05.

Key words and phrases. Lo-proximity, bunch, cluster. 
Let $(X, \delta)$ be a Lo-space. A collection $\sigma$ of subsets of $X$ is called a bunch provided $\sigma$ is nonempty and satisfies the following conditions:

(a) $A, B \in \sigma$ implies $(A, B) \in \delta$;

(b) $A \cup B \in \sigma$ if and only if $A \in \sigma$ or $B \in \sigma$;

(c) $\bar{A} \in \sigma$ implies $A \in \sigma$.

$\sigma$ is called a cluster if it is a bunch and satisfies the following:

(d) If $G \subset X$ and $(G, A) \in \delta$ for each $A \in \sigma$ then $G \in \sigma$.

A full account of this and other related material is given in [1].

Example 1. This is an example of a Lo-space in which some ultrafilters are contained in no cluster. Since each ultrafilter is contained in a maximal bunch, the Lo-space constructed here will contain maximal bunches which are not clusters.

Let $\mathcal{F}_{1}, \mathcal{F}_{2}, \mathcal{F}_{3}$ and $\mathcal{F}_{4}$ be four distinct nonprincipal ultrafilters on an infinite set $X$, and let $\mathcal{F}_{S}=\mathcal{F}_{1}$. Define a binary relation $\delta$ on the power set of $X$ as follows:

$A \delta B$ if and only if at least one of the following two conditions is satisfied:

(i) $A \cap B \neq \varnothing$;

(ii) For some $i, 1 \leq i \leq 4$, one of the sets $A, B$ is in $\mathcal{F}_{i}$ and the other belongs to $\mathfrak{F}_{i+1}$.

It is easy to verify that $\delta$ is a Lo-proximity. We claim that the filter $\mathcal{F}_{i}$ cannot be contained in any cluster for any $i, 1 \leq i \leq 4$. We prove this for the filter $\mathcal{F}_{1}$. If possible suppose there exists a cluster $\sigma$ such that $\mathcal{F}_{1} \subseteq \sigma$. Then $\mathcal{F}_{1} \subseteq \sigma \subseteq \mathcal{F}_{1} \cup \mathcal{F}_{2} \cup \mathcal{F}_{4}$. Take any $B \in \mathcal{F}_{2}$ and $C \in \mathcal{F}_{3}$ such that $C \notin \mathcal{F}_{1} \cup \mathcal{F}_{2} \cup \mathcal{F}_{4}$. Then $B \cup C \in \mathcal{F}_{2} \cap \mathcal{F}_{3}$. Therefore $(B \cup C) \delta P$ for each $P \in \mathcal{F}_{1} \cup \mathcal{F}_{2} \cup \mathcal{F}_{2}$. But since $\sigma \subseteq \mathcal{F}_{1} \cup \mathcal{F}_{2} \cup \mathcal{F}_{4},(B \cup C) \delta P$ for each $P \in \sigma$. As $\sigma$ is a cluster, we must have $B \cup C \in \sigma$. But as $C \notin \mathcal{F}_{1} \cup \mathcal{F}_{2} \cup$ $\mathcal{F}_{4}$, there exists $A \in \mathcal{F}_{1}$ such that $C \not A A$. Consequently $C \notin \sigma$, so $B \in \sigma$. Since $B \in \mathcal{F}_{2}$ was arbitrary, we have $\mathcal{F}_{2} \subseteq \sigma$. Similarly $\mathcal{F}_{4} \subseteq \sigma$, and thus $\sigma=\mathcal{F}_{1} \cup \mathcal{F}_{2} \cup \mathcal{F}_{4}$. Now take two sets $B$ and $D$ such that $B \cap D=\varnothing$, $B \in \mathfrak{F}_{2}, D \in \mathcal{F}_{4}, B \notin \mathcal{F}_{1} \cup \mathcal{F}_{3} \cup \mathcal{F}_{4}$ and $D \notin \mathcal{F}_{1} \cup \mathcal{F}_{2} \cup \mathcal{F}_{3}$. By our choice $B \not D D$ and both $B$ and $D$ belong to $\sigma$. This is a contradiction. Thus we conclude that there is no cluster containing the filter $\mathcal{F}_{1}$. The same is true for the filters $\mathcal{F}_{2}, \mathcal{F}_{3}$ and $\mathcal{F}_{4}$.

Example 2. This is an example of a Lo-space, in which, even though each ultrafilter is contained in a cluster, not every maximal bunch is a cluster.

Define a binary relation $\delta$ on the power set of the set $R$ of real numbers as follows:

$(A, B) \in \delta$ if and only if at least one of the following four conditions is satisfied.

(i) $A \cap B \neq \varnothing$. 
(ii) One of $A$ and $B$ contains an infinite subset of positive integers and the other contains an uncountable subset of positive real numbers.

(iii) One of $A$ and $B$ contains an infinite subset of negative integers and the other contains an uncountable subset of negative real numbers.

(iv) $A$ and $B$ are both uncountable.

The verification of $\delta$ being a separated Lo-proximity is straightforward. Also the collection $\zeta$ of all uncountable subsets of $R$ can easily be seen to be a bunch. We claim that $\zeta$ is a maximal bunch but not a cluster.

To show that $\zeta$ is a maximal bunch, take any bunch $\zeta_{1}$ such that $\zeta \subseteq \zeta_{1}$. It suffices to show that $\zeta=\zeta_{1}$. To see this let $A \in \zeta_{1}$. Write $A^{+}=$ $\{n \in A: n$ is a positive integer $\}, A^{-}=\{n \in A: n$ is a negative integer $\}$ and $B=\left\{x \in A: x \notin A^{+} \cup^{\prime} A^{-}\right\}$. Since the set $R^{-}$of all negative real numbers is in $\zeta$ and $\left(A^{+}, R^{-}\right) \notin \delta$, then $A^{+} \notin \zeta$. Similarly $A^{-} \notin \zeta$, and therefore $A^{+} \notin$ $\zeta_{1}$ and $A^{-} \notin \zeta_{1}$. Since $A \in \zeta_{1}$ and $A=A^{+} \cup A^{-} \cup B$, then $B \in \zeta_{1}$. Let $E$ be any uncountable subset of $R$. Then $E \in \zeta_{1}$ and therefore $(E, B) \in \delta$.

Since $B$ contains no positive integer nor any negative integers and $(E, B) \in \delta$ for any arbitrary uncountable subset $E$ of $R$, it follows from the definition of $\delta$ that $B$ is uncountable and, consequently, so is $A$. It follows that $A \in \zeta$ and, therefore, $\zeta=\zeta_{1}$. This proves that $\zeta$ is a maximal bunch. To show that $\zeta$ is not a cluster it is enough to observe that for the set $I$ of all integers we have $(I, A) \in \delta$ for each $A \in \zeta$, whereas $I \notin \zeta$.

Now we show that each ultrafilter on $(R, \delta)$ is contained in a cluster. Take any nonprincipal ultrafilter $\mathcal{F}$ on $R$. Then one of the sets $P=\{x \in R: x>0\}$ and $N=\{x \in R: x<0\}$ is in $\mathcal{F}$. Without any loss of generality, assume $P \in \mathcal{F}$. Let $I^{+}$be the set of all positive integers. At least one of the following three cases holds.

Case I. $I^{+} \in \mathcal{F}$. In this case the collection $\sigma=\{A \subseteq R$ : $A \in \mathcal{F}$ or $A$ contains an uncountable subset of $P\}$ is a cluster containing $\mathcal{F}$.

Case II. $I^{+} \notin \mathcal{F}$ and some member of $\mathcal{F}$ is countable. In this case $\mathcal{F}$ itself is a cluster.

Case III. Each member of $\mathcal{F}$ is uncountable. Let $\mathcal{G}$ be a nonprincipal ultrafilter on $I^{+}$. Then $\sigma=\{A \subset R: A \cap P$ is uncountable or $A$ contains some member of $\mathcal{G}\}$ is a cluster containing $\mathfrak{F}$.

Thus in all cases, $\mathcal{F}$ is contained in a cluster.

The author wishes to thank the referee for his valuable suggestions.

\section{REFERENCF}

1. S. A. Naimpally and B. D. Warrack, Proximity spaces, Cambridge Tracts in Math. and Math. Phys., no. 59, Cambridge Univ. Press, London and New York, 1970. MR $43 \# 3992$.

DEPARTMENT OF MATHEMATICS, SOUTHERN ILLINOIS UNIVERSITY, CARBONDALE, ILLINOIS 62901

Current address: Department of Mathematics, University of Kansas, Lawrence, Kansas 66045 